\title{
Anestesia para reparación antenatal de mielomeningocele: Presentación de un caso
}

\author{
Anesthesia for myelomenigocele antenatal repair: \\ Case report
}

María José Balanda Lira1, Marco Balkenhol Neumann²

\begin{abstract}
Introduction: The advance in the methods of prenatal diagnosis and surgical techniques have allowed the development of fetal surgery, achieving identification and early treatment of anomalies invalidating extrauterine life. Myelomeningocele (MMC) is the most frequent neural tube defect and its intrauterine correction has demonstrated benefits. Objective: To publicize the anesthetic management of a prenatal correction of MMC performed in a public hospital in Chile. Case report: 31-year-old woman, pregnancy of 25 weeks of gestational age, fetus carrying MMC lumbosacral, who underwent open correction. Procedure performed with incidents under general anesthesia with remifentanil and sevoflorane MAC in 2 and tocolytic prophylaxis. At 48 hours post operative, he presented an acute pulmonary edema compatible (EPA), which was successfully resolved with depletive therapy for 24 hours in the Intensive Care Unit, without the need for mechanical ventilation or use of vasoactive drugs. Discharged one week later in good condition, with interruption of pregnancy by elective caesarean section at 37 weeks, with a newborn without stigmas of neurological sequelae. Conclusions: The mother-fetus binomial is a challenge for the anesthetist. In intrauterine surgery the need for knowledge about the pharmacology of tocolytics, placental uterine physiology and the complications of the procedure are added.
\end{abstract}

\section{RESUMEN}

Introducción: El avance en los métodos de diagnóstico prenatal y las técnicas quirúrgicas han permitido el desarrollo de la cirugía fetal, logrando identifi-

\section{Key words:}

Total lung lavage, pulmonary alveolar proteinosis

\section{Palabras clave:}

Mielomeningocele, cirugía fetal, anestesia fetal, cirugía intrauterina

\footnotetext{
Anestesióloga Hospital Doctor Luis Tisné Brousse. Santiago, Chile.

2 Anestesiólogo Hospital Dr. Eduardo Schütz Schroeder. Puerto Montt, Chile.
}

Fecha de recepción: 19 de febrero de 2020

Fecha de aceptación: 14 de abril de 2020

\section{ORCID}

https://orcid.org/0000-0002-2057-2570

\section{Correspondencia:}

María José Balanda Lira

mjbalanda@gmail.com 
cación y tratamiento precoz de anomalías invalidantes para la vida extrauterina. El mielomeningocele (MMC) es el defecto del tubo neural más frecuente y su corrección intrauterina tiene beneficios demostrados. Objetivo: Dar a conocer el manejo anestésico de una corrección prenatal de MMC realizada en un hospital público de Chile. Caso clínico: Mujer de 31 años, embarazo de 25 semanas de edad gestacional, feto portador de MMC lumbosacro, que se sometió a una corrección por vía abierta. Procedimiento realizado con incidentes bajo anestesia general con remifentanilo y sevoflorano MAC en 2 y profilaxis tocolítica. A las 48 horas postoperatorias presentó cuadro compatible con edema pulmonar agudo (EPA), que se resolvió exitosamente con terapia depletiva por 24 horas en Unidad de Cuidados Intensivos, sin necesidad de ventilación mecánica ni uso de drogas vasoactivas. Dada de alta una semana después en buenas condiciones. El embarazo se interrumpió por cesárea electiva a las 37 semanas, con un recién nacido sin estigmas de secuela neurológica. Conclusiones: El binomio madre-feto es un reto para el anestesista. En cirugía intraútero se suma la necesidad de conocimientos sobre la farmacología de los tocolíticos, fisiología útero placentaria y las complicaciones propias del procedimiento.

\section{Introducción}

E $\mathrm{n}$ Chile, el mielomeningocele (MMC) es la segunda causa de malformación congénita[1],[2]. Se caracteriza por el cierre incompleto de la columna vertebral, dejando la médula expuesta al medio ambiente fetal[3]. El daño derivado del MMC es producido por el propio defecto del cierre y por la exposición prolongada del tejido nervioso al líquido amniótico y trauma directo por movimientos fetales[4]. Esto fundamenta que la reparación prenatal temprana de la lesión previene el daño y mejora el resultado clínico[5].

Dado que esta es una cirugía cada vez más difundida, con particularidades desde el punto de vista anestésico, se considera importante dar a conocer el manejo proporcionado en este caso.

\section{Caso clínico}

Paciente femenina, 31 años, sin antecedentes mórbidos, 2 cesáreas y 1 legrado uterino. Embarazo de 25 semanas con feto portador de MMC lumbosacro, diagnosticado en la semana 18 por ecotomografía obstétrica que evidencia signo del limón, cisterna magna obliterada y cerebelo con signo de la banana, asociado a un defecto de cierre de la columna lumbosacra L4-S1 y anomalía de Chiari secundaria. Cumple criterios de inclusión para cirugía antenatal[6], por lo que se planifica reparación abierta.

Plan anestésico: Anestesia general con sevoflo- rano y remifentanilo, monitorización estándar, línea arterial, profilaxis tocolítica y analgesia multimodal.

Preoperatorio: canalización de 2 accesos venosos y catéter urinario, profilaxis antibiótica con cefazolina $2 \mathrm{~g}$ y profilaxis tocolítica con indometacina $50 \mathrm{mg}$ rectal y sulfato de magnesio $\left(\mathrm{M}_{\mathrm{g}} \mathrm{SO}_{4}\right)$ en carga de $5 \mathrm{~g}$ e infusión a $1 \mathrm{~g} / \mathrm{h}$.

Intraoperatorio: pabellón preparado a $26^{\circ} \mathrm{C}$. Administración de ácido tranexámico $1 \mathrm{~g}$ y premedicación con midazolam 2 mg. Monitorización estándar, línea arterial, monitor de profundidad anestésica BIS, termómetro, monitor de bloqueo neuromuscular TOF, manta térmica BairHugger ${ }^{\mathrm{TM}} 3 \mathrm{M}^{\circledR}$ y colocación de catéter peridural \#16, L3-L4.

Inducción de secuencia rápida con lidocaína 20 $\mathrm{mg}$, remifentanilo $\mathrm{TCl}$, propofol $150 \mathrm{mg}$ en bolo, bloqueo neuromuscular con succinilcolina $80 \mathrm{mg}$ y rocuronio $30 \mathrm{mg}$. Intubación al primer intento con TOT No 7, laringoscopía directa Cormack G1. Mantenimiento de anestesia con remifentanilo y sevoflorano $4 \%-5 \%$ para MAC 1.5 y 2. Continuidad de $\mathrm{M}_{9} \mathrm{SO}_{4}$ en $\mathrm{BIC}$ y adición de nitroglicerina (NTG) en infusión a $5 \mathrm{mcg} /$ min. Analgesia con metamizol $2 \mathrm{~g}$ y paracetamol $1 \mathrm{~g}$ ev, profilaxis antiemética con dexametasona y ondansetrón 4 mg para ambos fármacos. Ventilación mecánica controlada por volumen, VT $400 \mathrm{ml}$, FR 15, PEEP 5 y $\mathrm{ETCO}_{2} 30-35 \mathrm{mmHg}$.

Intraoperatorio: paciente hemodinámicamente estable, latidos cardiofetales con un mínimo de 111 latidos por minuto que ocurrió durante manipulación quirúrgica del feto. Volumen $2.000 \mathrm{cc}$ de Ringer Lactato, diuresis 1.100 cc y sangrado de 200 cc. Procedi- 
miento completado en 4,5 h sin incidentes. Reversión del bloqueo neuromuscular con sugammadex 100 mg.

Postoperatorio: monitorización materno-fetal, indometacina $50 \mathrm{mg}$ día vr y progesterona $200 \mathrm{mg}$ cada 6 h vía vaginal, continuar con $\mathrm{Mg}_{\mathrm{g}} \mathrm{SO}_{4}$ y NTG. Analgesia con metamizol, paracetamol por horario y PCA peridural con bupivacaína 0,1\% + fentanilo 2 $\mathrm{mcg} / \mathrm{ml}$.

Postoperatorio inmediato paciente con dolor leve, episodios de dinámica uterina y tendencia a la hipotensión. Se adiciona tercer tocolítico atosivan, se disminuye dosis de PCA y se inicia fenilefrina a 0,1 $\mathrm{mcg} / \mathrm{k} / \mathrm{min}$. Transcurrido el primer día postquirúrgico se disminuye $\mathrm{M}_{\mathrm{g}} \mathrm{SO}_{4}$, se suspende NTG y ajusta PCA a demanda. En el segundo día postoperatorio paciente inicia paulatinamente desaturación hasta 90\% asociado a disnea y polipnea, sin otro compromiso hemodinámico. Examen físico compatible con edema pulmonar agudo. Se traslada a UTI donde estuvo 48 h con tratamiento depletivo, sin falla renal ni respiratoria que requiriera apoyo ventilatorio. Posterior a ello evolución favorable. Al séptimo día ecografía obstétrica sin signos de separación amniocorial y perfil fisiológico fetal normal. Es dada de alta manteniendo tocolíticos.

Se interrumpe el embarazo a las 37 semanas con cesárea electiva. A la fecha el paciente se encuentra sano, en controles y sin ninguna manifestación de daño neurológico.

\section{Discusión}

La etiología de los DTN está asociada a tres factores: a) Ambientales: con mayor presentación en la quinta, sexta y octava región de Chile, estrato socioeconómico bajo, ingesta de alcohol durante el embarazo y exposición a sustancias como ácido valproico, carbamazepina y plomo; b) Genéticos: asociación con trisonomía 13, 18 y 21 y mayor recurrencia entre hermanos. c) Nutricionales: demostrada asociación con el déficit de ácido fólico[1],[2].

Un 15\% de los pacientes no sobrevive sobre los 5 años y quienes lo hacen presentan secuelas de por vida con déficit motor y sensorial por debajo del nivel metamérico de la lesión, produciendo parálisis, disfunción intestinal, vesical y anomalías ortopédicas. La mortalidad es mayor con Arnold-Chiari II asociado, con una fosa posterior colapsada y el cerebelo herniado por el foramen magnun[7]. De estos pacientes, hasta $85 \%$ presenta hidrocefalia fetal que en la mayoría de los casos va a requerir de forma postnatal

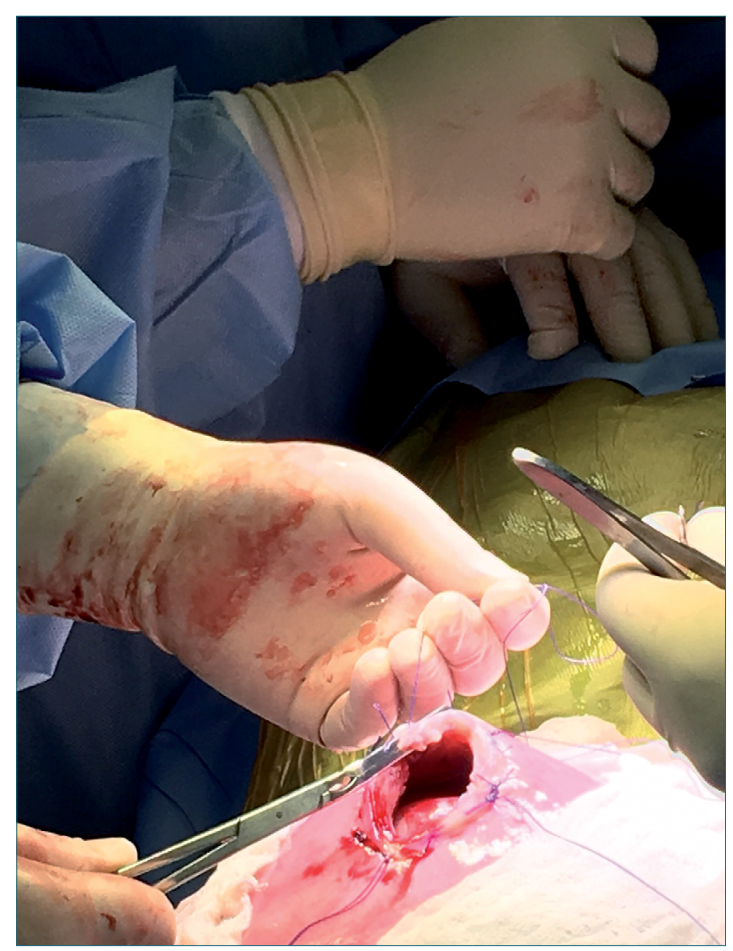

Figura 1. Equipo de neurocirugía realizando la reparación del MMC.

una derivación ventriculoperitoneal (DVP) ${ }^{8}$.

La corrección antenatal del MMC puede ser vía fetoscopía o por técnica abierta (Figura 1). El estudio MOMS (Management of Myelomeningocele Study) publicado en 2011 evidencia los beneficios de la cirugía fetal. Es un ensayo clínico aleatorizado multicéntrico que demostró que la cirugía prenatal abierta puede reducir a la mitad la necesidad de DVP en el primer año de vida, con mejor rendimiento intelectual y función motora a los 30 meses. Estos beneficios se asociaron a morbilidad materna importante (riesgo de sangrado y transfusión, edema pulmonar agudo, infección de la herida, dehiscencia de cicatriz uterina y necesidad de cesárea en gestación actual y sucesivas) y morbilidad fetal (corioamnionitis, parto prematuro y muerte fetal). La fetoscopía al ser menos invasiva se asocia a menores complicaciones maternas, pero con mayor tasa de parto prematuro y dehiscencia del sitio de reparación[9],[10].

Dado que la madre no tiene ningún beneficio directo por la intervención quirúrgica, el plan anestésico se basó en mantener el bienestar materno-fetal y disminuir las complicaciones maternas. Los objetivos fueron asegurar: oxigenación fetal, relajación uterina y analgesia postoperatoria. 
La hipoxemia fetal desencadena acidosis metabólica y muerte fetal. El flujo sanguíneo útero-placentario (FSUP) encargado del intercambio gaseoso con el feto, no tiene autorregulación y es dependiente de la presión arterial materna. Por ello es fundamental prevenir cualquier desencadenante de hipotensión, como el síndrome de hipotensión supina por la compresión aorto-cava, hipovolemia y el uso de fármacos cardiodepresores y vasodilatadores[11],[12],[13]. La hipoxemia prolongada y los cambios en la $\mathrm{paCO}_{2}$ materna también pueden alterar la oxigenación fetal. La hipocapnia produce vasoconstricción uterina y por tanto, disminución del FSUP. Por otro lado, la hipercapnia causa alcalosis materna y desplaza la curva de oxígeno-hemoglobina a la izquierda, reduciendo la extracción fetal de oxígeno. Se debe considerar que la $\mathrm{paCO}_{2}$ normal en la gestante es de unos 32 $\mathrm{mmHg}$, por lo que cifras consideradas ligeramente altas en pacientes no gestantes pueden significar una hipercapnia en la embarazada[14]. Se aseguró la oxigenación fetal con posición materna en tilt de $20^{\circ}$ a izquierda para disminuir la compresión aorto-cava, mantención de PAM adecuada con bolos de fenilefrina, $\mathrm{FiO}_{2}, 100 \%$ durante toda la cirugía, $\mathrm{ETCO}_{2}$ entre 30 y $35 \mathrm{mmHg}$ y mantención de normovolemia con ringer lactato, no superando los $2.000 \mathrm{cc}$ por riesgo de edema pulmonar agudo (EPA)[15].

La relajación uterina reduce el riesgo de parto prematuro que puede desencadenarse por la manipulación uterina, asegura el óptimo intercambio gaseoso placentario y disminuye el sangrado intraoperatorio. Halogenados con MAC sobre 2 producen relajación uterina, pero con predominio de los efectos cardiodepresores. Además, también se describe en la literatura el caso de crisis convulsiva en una paciente sometida a cirugía antenatal con MAC 2.5 con sevoflorano[16]. Se optó en el caso presentado por sevoflorano con MAC 1.5-2, complementado con infusión de $\mathrm{M}_{9} \mathrm{SO}_{4}$ y NTG, con lo que se obtuvo una excelente relajación uterina (Figuras 2 y 3 ).

La hipotermia fetal también causa acidosis y muerte, ya que el feto carece de termorregulación y además, pierde calor a través de su piel inmadura y del líquido amniótico[13]. Dado lo anterior se programó la temperatura de pabellón a $26{ }^{\circ} \mathrm{C}$, se cubrió a paciente con manta térmica y se utilizaron soluciones tibias endovenosas y para lavar.

Un manejo adecuado del dolor postoperatorio tiene un efecto tocolítico. La analgesia disminuye la liberación de catecolaminas, estradiol, cortisol y dehidroandrosterona, sustancias que favorecen las contracciones uterinas[17]. Como ya se describió, se utilizó analgesia multimodal endovenosa más PCA peridural.

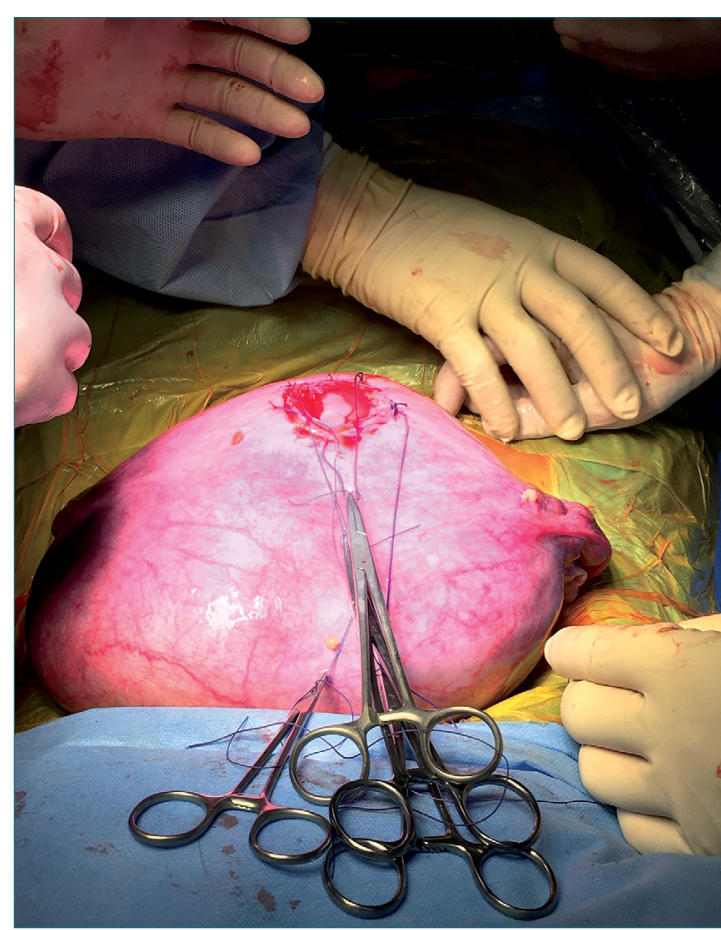

Figura 2. Realización de histerotomía, se puede apreciar la apropiada relajación uterina, ya que no hay salida de líquido amniótico a presión.

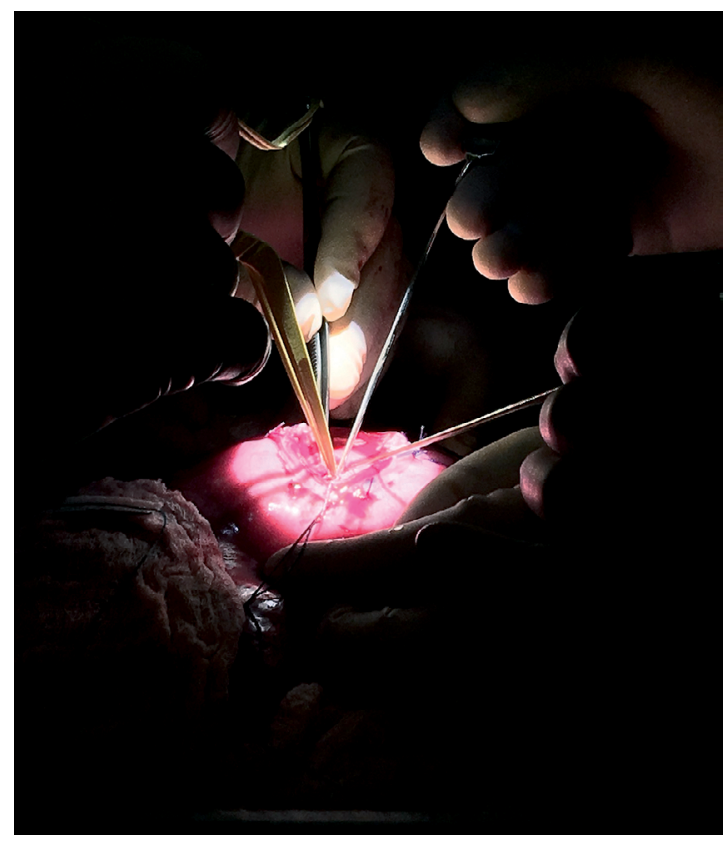

Figura 3. Imagen posterior a la corrección del MMC, útero completamente exteriorizado y relajado, previo al inicio de la histerorrafia. 
Una complicación materna descrita en el MOMS y en múltiples metaanálisis es el EPA[4],[18],[19]. Su patogenia es secundaria a distintas variables: las gestantes tienen presión coloido oncótica disminuida, mayor permeabilidad capilar y un eje renina angiotensina aldoesterona más activo, adicionalmente la cirugía desencadena liberación de prostaglandinas que favorecen el edema intersticial y se utilizan fármacos como el $\mathrm{M}_{\mathrm{g}} \mathrm{SO}_{4}$ que causa vasodilatación y la NTG que es precursor de peroxinitrito que daña el neumocito tipo II y causa injuria pulmonar ${ }^{18}$. La paciente del caso descrito presentó EPA a pesar del volumen restrictivo y control del balance hídrico. Mirándolo retrospectivamente, una ecografía pulmonar seriada podría detectar un pulmón congestivo previo a los síntomas. rúrgicas apuntan a aumentar los resultados neonatales, el peligro para la salud materna persiste como un tema crucial. Hay que adaptar la anestesia a los cambios anatomofisiológicos de la gestación y enfocarse en prevenir y diagnosticar precozmente las complicaciones.

Para conseguir la máxima seguridad del binomio materno-fetal, idealmente la cirugía debería limitarse a centros con un equipo de expertos que se reúnan previo al procedimiento y mantengan una comunicación constante, a modo de planificar toda la conducta terapéutica.

Cirugías complejas y mutidisciplinarias refuerzan la necesidad de la medicina perioperatoria, ya que es necesario un manejo integral, continuo y lineal desde el preoperatorio hasta el alta.

\section{Conflicto de interés}

La autora declara que no existe conflicto de intereses.
Aunque los esfuerzos de un mayor avance de la reparación prenatal de MMC con nuevas técnicas qui-

\section{Referencias}

1. Nazer J, Cifuentes L. Resultados del Programa de Prevención de Defectos de Tubo Neural en Chile mediante la fortificación de la harina con ácido fólico. Período 2001-2010. Rev Med Chil 2013;141(6):751-7. https://doi.org/10.4067/S003498872013000600009 .

2. Devoto JC, G, Tapia M. Cirugía prenatal del mielomeningocele: Un nuevo desafío. Rev Chil Anest 2016; 45: 6-15. https:// doi.org/10.25237/revchilanestv45n01.01.

3. Adzick NS. Fetal myelomeningocele: natural history, pathophysiology, and in-utero intervention. Semin Fetal Neonatal Med. 2010 Feb; 15(1): 9-14. https://doi.org/10.1016/j. siny.2009.05.002.

4. Adzick NS, Thom EA, Spong CY, Brock JW, Burrows PK, Johnson MP y cols. A randomized trial of prenatal versus postnatal repair of myelomeningocele. N Engl J Med. 2011; 364(11):993-1004. https://doi.org/10.1056/NEJMoa1014379.

5. Keller BA, Farmer DL. Fetal surgery for myelomeningocele: history, research, clinical trials, and future directions. Minerva Pediatr. 2015 August; 67(4): 341-56.

6. Otayza Felipe, Cirugía Fetal del Mielomeningocele. Rev. Medica Clínica Las Condes - 2015; 26(4) 442-451. https://doi.org/10.1016/j.rmclc.2015.07.003.

7. Bowman RM, McLone DG, Grant JA, Tomita T, Ito JA. Spina bífida outcome: A 25-year prospective. PediatricNeurosurg. 2001; 34: 114-120. https://doi. org/10.1159/000056005.

8. Caldarelli M, Di Rocco C, La Marca F. Shunt complications in the first postoperative year in children with meningomyelocele. Childs Nerv Syst. 1996; 12(12):748-54. https://doi. org/10.1007/bf00261592.

9. Sacco A, Van der Veeken L, Bagshaw E y cols. Maternal complications following open and fetoscopic fetal surgery: A systematic review and meta analysis. Prenatal Diagnosis. 2019; 39: 251-268. https://doi. org/10.1002/pd.5421

10. Kabagambe $S$, Jensen $G$, Chen $Y$, Vanover M, Farmer D. Fetal Surgery for Myelomeningocele: A Systematic Review and Meta-Analysis of Outcomes in Fetoscopic versus Open Repair. Fetal DiagnTher 2018; 43:161-174. https://doi.org: 10.1159/000479505

11. Cheek TG, Baird E. Anesthesia for nonobstetric surgery: maternal and fetal considerations. ClinObstet Gynecol. 2009 Dec; 52(4): 535-45. https://doi.org/10.1097/ GRF.0b013e3181c11f60.

12. Tran K, Smiley R, Schwartz AJ. Anesthesia for fetal surgery: miles to go before 
we sleep. Anesthesiology. 2013 Apr; 118 (4): 772-4. https://doi.org/10.1097/ ALN.0b013e318283c976.

13. Sviggum HP, Kodali BS. Maternal anesthesia for fetal surgery.ClinPerinatol. 2013 Sep; 40 (3): 41327. https://doi.org/10.1016/j. clp.2013.05.012.

14. Monica A. Hoagland \& Debnath Chatterjee. Anesthesia for fetal surgery. Pediatric Anesthesia 27 (2017) 346-357. https://doi. org/10.1111/pan.13109.

15. DiFederico EM, Burlingame JM, Kilpatrick SJ, Harrison M, Matthay MA. Pulmonary edema in obstetric patients is rapidly resolved except in the presence of infection or of nitroglycerin tocolysis after open fetal surgery. Am J ObstetGynecol 1998;179(4):925-33. http:/ doi.org/10.1016/S00029378(98)70190-5.

16. C. Shavit, M. Rollins and M. Ferschl.Maternal convulsion during high-dose sevofluraneanaesthesia for open foetal surgery. British Journal of Anaesthesia, Volume 118, Issue 4, April 2017, Pages 634-635. https://doi. org/10.1093/bja/aex067.

17. Wu D, Ball RH. The maternal side of Maternal-Fetal Surgery.ClinPerinatol. 2009 Jun; 36(2): 247-
53. https://doi.org /10.1016/j. clp.2009.03.012.

18. Licci M, Guzman R, MD, Soleman J. Maternal and obstetric complications in fetal surgery for prenatal myelomeningocele repair: a systematic review. NeurosurgFocus. Oct 2019; 47(4)https://doi.org/ 10.3171/2019.7.FOCUS19470.

19. Etchegaray A, Palma F, De Rosa $R$ y cols. Cirugía fetal de mielomeningocele: Evolución obstétrica y resultados perinatales a corto plazo de una cohorte de 21 casos. SurgNeurollnt. 2018; 9(4):73-84. https://doi.org/ 10.4103/sni.sni_236_18. 